\title{
The e-POP Radio Receiver Instrument on CASSIOPE
}

\author{
H.G. James ${ }^{1}$ - E.P. King ${ }^{1}$ - A. White ${ }^{1}$ R.H. Hum ${ }^{1}$. \\ W.H.H.L. Lunscher ${ }^{2}$ C.L. Siefring ${ }^{3}$
}

Received: 6 March 2014 / Accepted: 17 December 2014 / Published online: 30 January 2015

(C) The Author(s) 2015. This article is published with open access at Springerlink.com

\begin{abstract}
The Radio Receiver Instrument (RRI) is a four-channel digital receiver fed by four 3-metre monopoles, arranged in a crossed configuration, each connected to a high input impedance preamplifier. The RRI bandwidth extends from $10 \mathrm{~Hz}$ to $18 \mathrm{MHz}$. The receiver measures the electric fields of either spontaneous radio emissions or waves created by ground transmitters, such as ionosondes, high-frequency radars and ionospheric heaters. In order to measure accurately the intensity, frequency, direction of propagation and signal delay of such fields over the broad frequency range, modern digital receiver technology is employed. The amplified signals from the monopoles are digitized at a rate of 40 megasamples per second, and from there on, the signal is down-converted, filtered, time-stamped and communicated in digital form. The characterization results of the RRI flight model are reported. Formats for data commands for configuring the digital receiver and for data output are described.
\end{abstract}

Keywords Ionosphere · Electric fields · Wave propagation · Digital receiver · Signal processing

\section{Introduction}

The Radio Receiver Instrument (RRI) has been launched on the Canadian Smallsat CASSIOPE as part of the enhanced Polar Outflow Probe (e-POP) mission (Yau and James 2011). The RRI is designed to exploit recent advances in digital broadband radio techniques for research topics in space radio science. The science objectives are summarized in the present section, which is followed in Sect. 2 by a description of the major functional components

\footnotetext{
$凶$ H.G. James

james@phys.ucalgary.ca

1 University of Calgary, Calgary, T2N 1N4, Canada

2 COMDEV Canada, Ottawa, K2K 3J1, Canada

3 Naval Research Laboratory, Washington, 20375, USA
} 
of the RRI. Section 3 summarizes the characterization of the instrument, and illustrates the flexibility of the instrument by providing the input parameters that define the operating configurations of this software-controlled radio. Section 4 provides some examples of the formats of the resulting data files based on prelaunch measurements. In Sect. 5, we include transmissions received in flight from the SPEAR ionospheric heater in Svalbard, Norway, to show that the RRI is functioning as designed.

The present paper follows earlier articles on RRI written during its design (James et al. 2002; James 2006a). The scientific objectives of e-POP/RRI have not changed significantly. Hence this paper concentrates on the details of the engineering design that has been followed to meet the scientific requirements laid out in 2002. Section 3 is a synopsis of a Radio Receiver Instrument Characterization Report that was submitted by some of the authors to the e-POP Project Office on 8 March 2010 and that is held there as an intra-project reference document for interested users.

\subsection{Experimental Objectives}

The RRI is a four-channel digital receiver fed by four 3-metre monopoles, arranged in a crossed configuration, each connected to a high input impedance preamplifier. The RRI total bandwidth extends from $10 \mathrm{~Hz}$ to $18 \mathrm{MHz}$. The receiver measures the electric fields of either spontaneous radio emissions or waves created by ground transmitters, such as ionosondes, high-frequency (HF) radars and ionospheric heaters. In order to accurately measure the intensity, frequency, direction of propagation and signal delay of such fields over the broad frequency range, modern digital receiver technology is employed. The amplified signals from the monopoles are digitized at a rate of 40 megasamples per second, and from there on, the signal is down-converted, filtered, time-stamped and communicated purely in digital form. For a description of the RRI design goals, and of the scientific objectives that drove them, the reader is referred to earlier papers by James et al. (2002) and James (2006a).

The scientific objectives of the RRI program are to improve understanding in the following areas:

\subsubsection{The Morphology and Dynamics of Density Structure in the Ionosphere}

The formation of density structures of a variety of sizes in the auroral regions is one of the important manifestations of the injection into the earth's atmosphere of energy and momentum originating at the sun. Fluid processes around the peak of the ionospheric F region, such as the gradient-drift instability, give rise to density irregularities. Joule heating may produce travelling ionospheric disturbances. Polar-cap patches are formed near the ionospheric cleft as a result of magnetospheric flux transfer. This part of the RRI agenda encompasses the detailed explanation of the physical origins of such structure and its subsequent motion through the ionosphere.

It is planned to observe waves from HF transmitters on the ground using the RRI on ePOP when it makes an orbital pass through the nearby topside ionosphere. The objective is to measure 4 basic quantities of the waves: the magnitude of the electric field $\mathbf{E}$, the Doppler frequency shift, the direction of arrival (DOA) and the signal-delay time. These four can all be applied to the "imaging" of ionospheric structures that produce the backscattered or reflected signals observed at those ground facilities. Such measurements are to be coordinated with simultaneous recordings at the ground facilities. 


\subsubsection{The Generation of Spontaneous Radio Emissions Created by Auroral Processes}

The 325-to-1500-km altitude range of the CASSIOPE spacecraft brings e-POP into the observation region of various wave-particle interactions (WPI) that give rise to freely propagating electromagnetic waves. The microphysical explanation of the exchange of energy between charged particles and electromagnetic (EM) waves can be addressed with the emphasis on processes involving energies that the e-POP Suprathermal Electron Instrument (SEI) and the Imaging Rapid Mass Spectrometer (IRM) can observe, that is, from thermal to about $100 \mathrm{eV}$. The WPIs that lead to up-flowing ion conics and transversely-accelerated ions are to be investigated with the RRI set to detect waves in the Ultra Low Frequency to Very Low Frequency range. Broadband ion cyclotron waves and lower-hybrid resonance waves are in this category. An explanation is sought for the trapping of intense lower-hybrid waves in density depletion cavities of 100-m extent. In the medium- to high-frequency range, investigations will be carried out on complicated auroral-latitude mechanisms capable of triggering electrostatic wave growth that eventually converts to cold-plasma wave modes that are observable on the ground. These F-layer emissions include such phenomena as "auroral roar" and "impulsive broadband emissions".

\subsubsection{The Nonlinear Plasma Physics of the HF-Modified Ionosphere}

In ionospheric modification experiments, HF waves are used to perturb the ionosphere. In the $\mathrm{E}$ region, the active change of plasma bulk properties through heating permits a controlled study of auroral electrojet-related processes. In the F region, stimulation of parametric instabilities provides insight into the role of nonlinearities in limiting microphysical plasma processes that influence bulk plasma characteristics. In previous experiments with the heater at Arecibo, Puerto Rico, wave fields with fine scale structures, both directly from the heater and excited nonlinearly in-situ, were observed. The coordinated use of RRI with ionospheric heaters is to elucidate the complex interplay between various electromagnetic (EM) and electrostatic wave modes and field-aligned density structures.

\subsubsection{The Physics and Metrology of Radio-Wave Scattering, Diffraction and Refraction}

The high-latitude ionosphere can have a dramatic effect on EM waves passing through it. Waves can be refracted, scattered, amplified, damped or nonlinearly decomposed, depending on the local state of the medium. The results from the OEDIPUS-C sounding-rocket mission illustrate the importance and efficiency of field-aligned ionospheric density structure in trapping and guiding HF EM waves (James 2006b). This structure may be the same as irregularities that coherently backscatter HF waves or those that cause spread-F in ionograms. Transionospheric propagation in e-POP exploits the attractions of two-point radio propagation experiments for better comprehension of the roles of scattering, diffraction and refraction.

These investigations of large-scale density structure, mentioned first above, are to be coordinated with searches for (oblique) backscatter from the small-scale irregularities.

\subsection{Quantities to Be Measured}

The RRI measures and records variables that define RF wave electric fields $\mathbf{E}$ incident upon the spacecraft. The magnitudes of $\mathbf{E}$ in the case of discrete modulated $\mathrm{CW}$ signals emitted 
by artificial sources on the ground below need to be accompanied by information about the wave polarization or DOA of the incident waves. As explained by James (2003), the DOA should be determinable in the case of identifiable HF ground transmitters detected in upper branch O and X modes of cold-plasma propagation (Budden 1985). We plan to determine DOA by using the magnitude and relative phase of voltages induced on the RRI monopoles combined as orthogonal dipoles.

A third measured parameter, the Doppler Shift of artificial signal carrier frequency, provides a consistency check on the wave DOA. Doppler shift is largely caused by orbital movement of the receiver with respect to the ionospheric medium through which it passes. The order of magnitude of such shift at HF may be as large as $\pm 100 \mathrm{~Hz}$. e-POP collaborates with scientific transmitters whose carrier frequencies are well controlled for measurements of this order. If the ionospheric plasma is in motion with respect to earth, then it is necessary to take this into account when computing the wave frequency as seen by the orbiting spacecraft. Doppler shifts caused by convective motion of the plasma are expected to be small compared with those caused by orbital motion.

A fourth quantity is absolute delay of signal from the instant of emission at the source. The insertion of time stamps into the received data stream is made with a sampling-rate precision of $\pm 8 \mu$ s by connection to the spacecraft clock, which is controlled by 1-pps ticks from the Global Positioning System (GPS) receivers in the GPS-based Attitude and Position (GAP) instrument in the e-POP payload. Determination of the absolute signal delay time of waves from the ground is thus limited by the RRI sampling rate, not by the superior timing of the GPS 1-pps. It is anticipated that the timing of pulses from all collaborating ground transmitters will have the GPS timing accuracy.

In the case of observation of spectra of spontaneously emitted EM waves arising from wave-particle interactions, the $\mathbf{E}$ field spectrum $E(f)$ is thus an important representation of observed data. Spectra are to be observed in particular characteristic frequency ranges, typically defined with respect to the electron plasma frequency, $f_{p e}$, and the electron gyrofrequency, $f_{c e}$.

\section{Instrument Description}

\subsection{Overview}

The purpose of this section is to set out the functional objectives of the RRI concept. Its design targets a dynamic range of $120 \mathrm{~dB}$ above an input threshold of $0.3 \mu \mathrm{V} \mathrm{rms}$. This total range is achieved by providing three gain settings, each offering a $72 \mathrm{~dB}$ dynamic range overlapping with the others. Full-scale input sensitivity is $1.26,39.8$, or $355 \mathrm{mV}_{\mathrm{RMS}}$ depending on the range. Both the amplitude and relative phase of an incoming electric field can be measured. After suitable amplification and low-pass filtering for anti-aliasing, the signals are digitized and then processed by a digital down converter and decimating lowpass filter. Receiver pass bandwidths of about $10 \mathrm{kHz}$ and $30 \mathrm{kHz}$ are programmable in each of the four receiver channels. The resultant digital data are time-tagged to an accuracy of better than $\pm 8 \mu \mathrm{s}$.

Figure 1 shows the $+x$ face of the CASSIOPE spacecraft with the four RRI monopoles deployed.

Figure 2 shows the functional blocks comprising the RRI system. The system has four identical signal processing channels, of which two are shown. Each channel is tied to a single monopole antenna. However, using a programmable relay, opposing monopoles can be 
Fig. 1 RRI monopole antennas on the CASSIOPE spacecraft

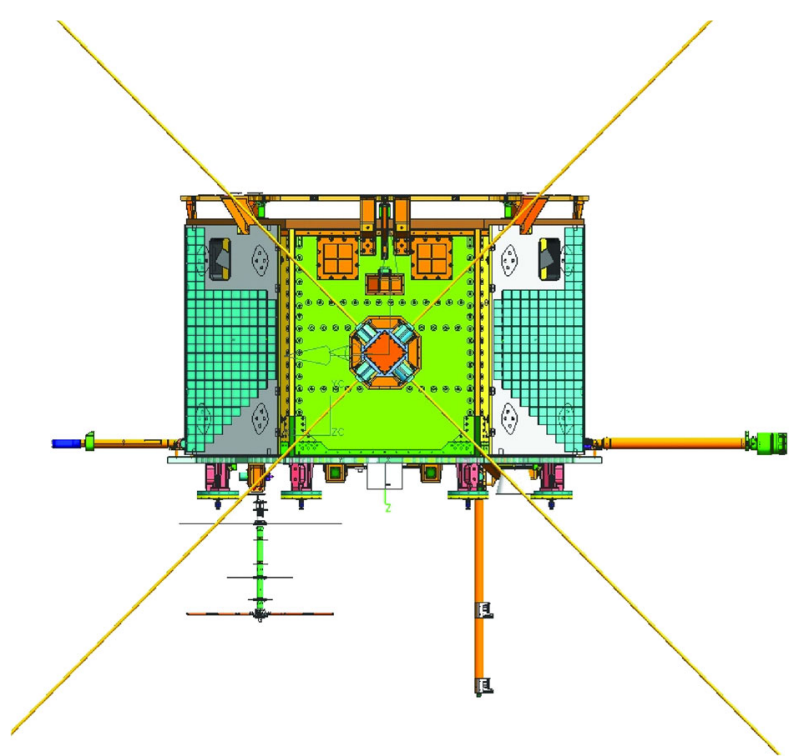

configured into a differential operation mode. Additionally, there is a power supply function providing the necessary voltage, current, and electromagnetic interference filtering for the electronic components, and a digital clock function required by the synchronous digital electronics. The following description treats the RRI as a system composed of three physically separate units: the antennas, the preamplifiers and a digital radio receiver module. These three separate functional units are identified with three background colors.

\subsection{Monopole Antennas, Supports and Preamplifier Enclosure}

Monopole antennas detect the electric field of electromagnetic waves at the CASSIOPE spacecraft. Each antenna is not intended to extract electromagnetic energy in the manner of an antenna impedance-matched to the input of a classic radio receiver; rather, the antenna acts as a high-impedance voltage probe of the wave environment. In this passive antenna arrangement, the goal is to relate voltage appearing at the antenna terminals directly to the amplitude of the wave electric field in the surrounding medium.

The monopoles are storable extendible members of $\mathrm{BeCu}$ forming a tubular structure $1.2 \mathrm{~cm}$ in diameter and $3 \mathrm{~m}$ long when released. Before launch these antennas are coiled up inside Delrin ${ }^{\circledR}$ insulating containers that are part of the antenna assembly. When an electrical signal is received from the spacecraft Data Handling Unit, a retaining door is released and the antenna's stored mechanical energy deploys the $\mathrm{BeCu}$ elements to their full length. The tubular antennas are 3-m "STEM Jib" monopoles manufactured by Astro Aerospace, and have considerable flight heritage. For reference to later discussion, the monopole to the upper right in Fig. 1 is no. 1, and no. 2 is at the lower left. Monopole 3 is at the upper left.

Figure 3 shows the major features of the $\mathrm{BeCu}$ tubular monopoles in relation to their Delrin ${ }^{\circledR}$ deployer housings, the support brackets and the PA enclosure. Such a design realizes the objective of bringing the inboard ends of the tubes as close as possible to the points where they electrically connect to their respective PA inputs inside the PA enclosure.

The antennas deploy under the energy of storage imparted to them when they are placed in the Delrin ${ }^{\circledR}$ deployers. A non-pyrotechnic actuator releases spring-loaded doors on the front face of the deployers. In turn, the $\mathrm{BeCu}$ elements deploy under their storage energy. 


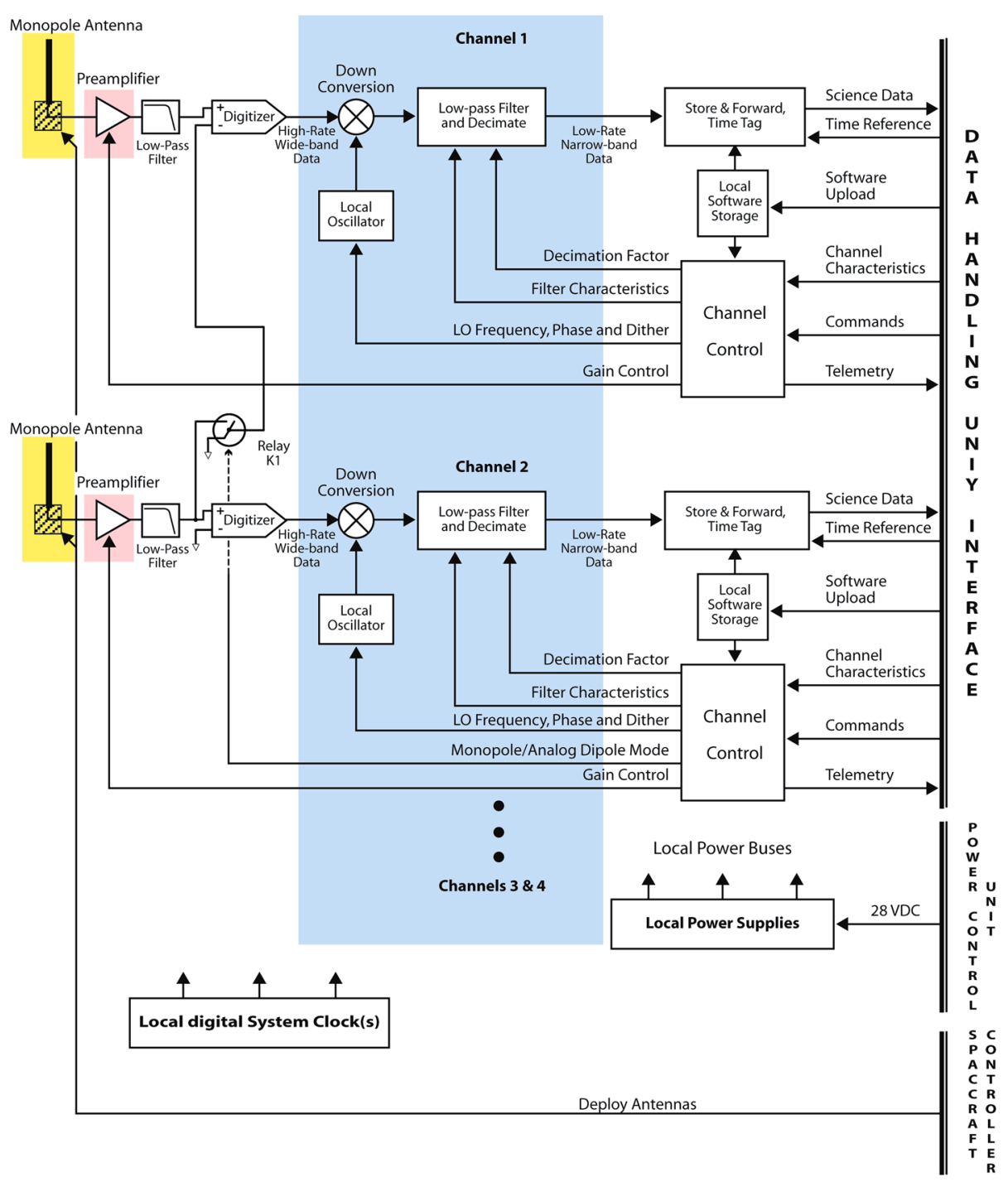

Fig. 2 Top-level functional block diagram of two of the four RRI channels

Views of the spacecraft with the RRI monopoles and all other instrument probes deployed are in Figs. 1 and 4. Instrument positions can be defined with respect to 3 orthogonal axes $x, y$, and $z$. The $+y$ axis is parallel to the direction along the SEI boom toward its detector head, while the $+z$ axis is parallel to the direction along the MGF boom toward the magnetometer modules. The orthogonal RRI monopoles lie in a plane parallel to the $y-z$ plane, and slightly above the face of the CASSIOPE spacecraft through which the $+x$ axis passes. CASSIOPE has an attitude control system that permits the spacecraft to take one of several different orientations, including one that slews fast enough to keep a direction in $x-y-z$ fixed on a target of constant latitude, longitude and altitude in earth-centered coor- 
Fig. 3 Aluminum preamplifier enclosure and supports (yellow) for monopole deployers (white)
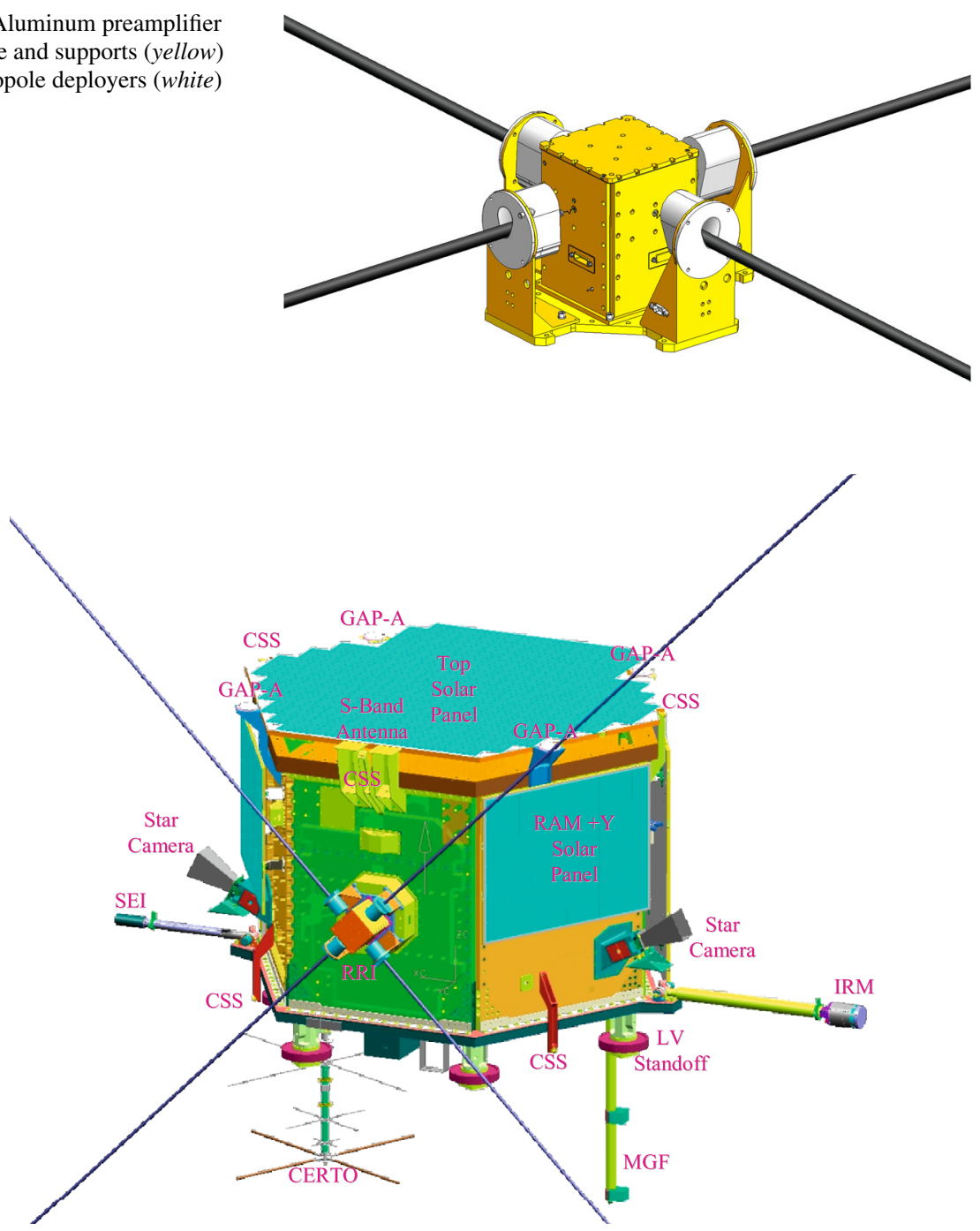

Fig. 4 Four 3-m RRI monopoles deployed on the $+x$ face of the CASSIOPE spacecraft, and other instruments of the e-POP payload (Yau and James 2011)

dinates. In the spacecraft orientation most frequently used, the $+x$ axis lies parallel to the spacecraft velocity direction, while the $+z$ axis maintains nadir direction.

\subsection{Preamplifiers}

The expected voltages induced on the antennas by the electrical fields of waves lie in a wide dynamic range (at least $120 \mathrm{~dB}$ ), in the low end requiring amplification by the preamplifier (PA) and, at the high end, attenuation. The PA must also present a high input impedance to the monopole antenna to assure that the input voltage to the preamplifier is the open-circuit value. These requirements were met with the PA schematic design in Fig. 5. 


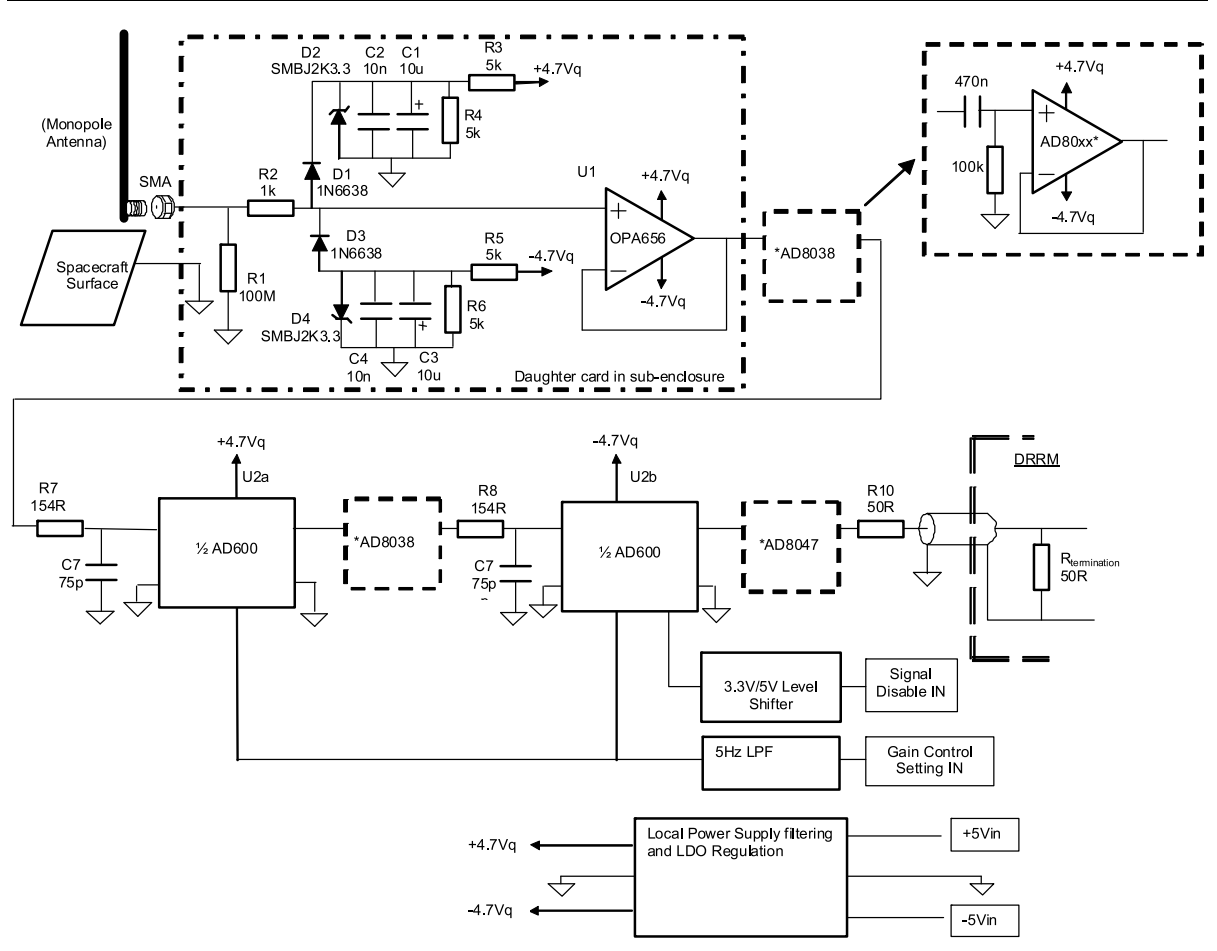

Fig. 5 Preamplifier simplified schematic

The range of expected input voltages requires a means of adjusting the gain of the PA to match the expected signal amplitude, so that the output signal exploits the linear dynamic range of the following digitizer block. Hence, the PA in Figs. 2 and 5 is shown accepting a gain control input command. The gain is set by command control along with the other RRI programmable parameters listed in Sect. 2.7. It was decided to operate the RRI with three PA gain settings of about $-11,+19$, and $+49 \mathrm{~dB}$, called "Low", "Medium", and "High", respectively.

Each monopole STEM Jib antenna is fitted with an SMA jack connecting the antenna to a preamplifier card via a short length of ETFE-insulated stranded wire fitted with a mating SMA plug. The electrical reference for the monopole is the spacecraft structure. This structure connects to the preamp signal ground through RF-quality electrical bonds to the preamplifier enclosure. The predicted source impedance of the monopole in the plasma environment resembles the impedance of a $160-\mathrm{pF}$ capacitor. In order to avoid significant signal attenuation, the preamplifier input impedance must be significantly higher than the source impedance. At HF, the input impedance is dominated by stray capacitance within the input circuit. Consequently, the shunt capacitance at the preamplifier input must be minimized.

In order to protect against damaging over-voltages (over $5 \mathrm{~V}$ sustained, or over $2 \mathrm{kV}$ transient) each preamplifier is equipped with an input over-voltage protection circuit at the monopole interface. This protection is provided by a low-capacitance, low-leakage steering diode tied to a transient voltage suppressor diode for electrostatic discharge protection, and a DC voltage source for signal clamping. To accommodate both positive and negative polarities, two circuits are required. Approximately $3 \mathrm{pF}$ of the total 11-pF input capacitance is estimated to be due to the protection circuit. 
An OPA656 input buffer serves as the interface between the monopole and the signal processing circuits. The noise floor of the entire preamplifier is dominated by noise at this front-end stage. So minimizing first stage noise sources has been a serious design consideration. One method employed to minimize noise is to encapsulate each OPA656 together with the input protection circuit within its own mini-enclosure fastened to the preamplifier enclosure face plates. The wideband (150-MHz measurement bandwidth) noise observed at the preamplifier output was approximately $140 \mathrm{mVpp}$ at the high gain setting and $15 \mathrm{mVpp}$ at the medium gain setting (150-MHz bandwidth, engineering model). With the application of the gain values defined above these measurements correlate to input-referred levels of $0.5 \mathrm{mVpp}$ and $1.68 \mathrm{mVpp}$ respectively, again in a 150-MHz bandwidth. The effective noise level at the radio output, as opposed to preamplifier output, is obviously much lower due to the application of narrow band filters in the subsequent digital radio circuits.

The OPA656 input buffer is followed by two variable-gain amplifiers connected in series, based on a single Analog Devices Inc AD600. The AD600 was chosen because it can provide 0 to $80 \mathrm{~dB}$ voltage gain with a single control voltage, DC-35 MHz bandwidth independent of gain, stable group delay, low noise and distortion, high output drive capability, and is specified for operation down to $-25^{\circ} \mathrm{C}$. However, its low input impedance of $100 \mathrm{ohms}$ ultimately became a bit of a drawback for RRI due to the need for $10-\mathrm{Hz}$ signal processing. When programmed for high gain, any significant DC offsets applied to the AD600 could give rise to signal clipping or other saturation effects at the output. Therefore DC blocking capacitors are required at the AD600 inputs. The 100-ohm input resistance leads to very large capacitor values (i.e. tantalum types) for LF signal handling. Worse still, back-to-back capacitors forming non-polar configurations are required to withstand the possibility of plus or minus rail transient or sustained output excursions caused by potential radiation effects. Aside from being physically large, such configurations were found to be a factor in undesired in-circuit HF oscillations and a contributor to response drift with temperature at low frequency. The solution to these issues was the addition of unity gain buffers between stages, the AD8038 and AD8047 circuit blocks shown in Fig. 5. The high input impedance of the buffers permits the use of sensibly sized $0.47 \mu \mathrm{F}$ BX dielectric capacitors, resolving all the aforementioned issues at the expense of slightly increased power consumption and slightly increased noise.

The AD8038 and AD8047 are both commercial, radiation-tolerant devices. Aside from not degrading electrical performance, the addition of these devices must have minimal impact on the power budget. Consequently two different parts are specified. In essence the AD8038 possesses all of the desired qualities (low power consumption (quiescent current $\approx 1 \mathrm{~mA}$ ), wide bandwidth, low noise and distortion) except for output drive capability. The AD8047 has the appropriate output stage to drive the $50 \mathrm{ohm}$ line to the DRRM, albeit at the price of much higher power consumption (quiescent current $\approx 7 \mathrm{~mA}$ ) and higher noise.

The gain control signals for the AD600 variable gain amplifiers are generated under DSP software control by an AD5327 12-bit digital-to-analog converter (DAC) on the Digital Radio Receiver card. This gain control signal is brought to each preamplifier card over a double-shielded cable and is subsequently filtered by inline ferrites and RC networks. Three gain settings were base-lined that result in the previously mentioned "low", "medium" and "high" overall system gains. An advantage of using a DAC for gain control is that these values can be precisely tuned and calibrated for each of the four cards.

The preamplifier bandwidth is deliberately limited so that out-of-band signals are not passed to the Digital Radio Receiver causing aliasing of the digitized signal. In combination, the three DC-blocking stages also act as third-order high-pass filters with a measured $-3 \mathrm{~dB}$ low frequency cut-off at $7 \mathrm{~Hz}$. The HF cut-off is also determined by a third-order filter 
implemented in the two AD600 variable gain stages with a measured $-3 \mathrm{~dB}$ frequency of $16 \mathrm{MHz}$. This 3-dB point is little lower than the desired $18 \mathrm{MHz}$, but this reduced bandwidth can be compensated in post processing.

\subsection{Digital Radio Receiver Module (DRRM)}

This section covers all the remaining functions in Fig. 2, that is, the functions to the right of the pink-colored PAs.

Digital radio techniques have been applied to the design of the RRI. The output of the PA is presented to a digitizer to convert the analog signal into a digital data stream. During instrument calibration, additional anti-aliasing low-pass filtering was found to be needed in advance of the digitizer to adequately band-limit the signal, ideally to half the digitizer rate. The specified total signal bandwidth is $18 \mathrm{MHz}$, therefore the digitizer must operate at a minimum sample rate of $36 \mathrm{MHz}$ requiring an anti-aliasing filter with an $18 \mathrm{MHz}$ bandwidth. A somewhat higher sampling rate, $40 \mathrm{MHz}$, has been chosen, to permit the filter to have a feasible high frequency roll-off. More detail about this low-pass anti-aliasing filtering, achieved with five-pole Butterworth filters, is found in Sect. 3.3 below.

An alternate path of the filtered data is through the indicated differential comparison block. This block is switched in when opposing monopoles are combined in a dipole mode of operation. When switched in, a differential amplifier, integrated with the digitizer, accepts the signals from two monopoles to produce an analog differential output signal that is presented to the digitizer instead of a monopole signal. A relay "K1" is shown toggling between the monopole and dipole connections. A differencing of digital monopole values can also be performed later during ground data processing, if required. Performing the operation in this way does not lose the individual monopole signal data, which may also be of interest. The output of the differential blocks is shown feeding into channel 1 only as a matter of convenience to simplify the diagram. In summary, monopole-1 voltage minus monopole- 2 voltage appears in output channel 1 and monopole- 3 voltage minus monopole- 4 voltage is output in channel 3.

The digitized signal is then mixed, or multiplied, with that of a stable, well-controlled local oscillator (LO), to move the signal pass band of interest down to the baseband region centered on DC. The frequency of the LO is programmable and changed according to the programmed plan for the current experiment. It is also possible to precisely program and synchronize the LO phase across the four channels. The LO mixer is a complex-number device mixing the signal with two sine waves having a $90^{\circ}$ phase difference producing an in-phase (I) and a quadrature (Q) signal output. These two I and Q signal trains are processed in parallel throughout the rest of the system.

The mixed signal is next filtered by the low-pass decimating filter block. The low pass filter aspect simply rejects the out-of band high-frequency signal components. Since a finite impulse response (FIR) filter is employed in this filter block, it is also readily capable of other, more sophisticated operations such as matched filtering. The decimating aspect modifies the output sample rate to better match the reduced signal bandwidth. For instance, if the analog signal is sampled at a $40 \mathrm{MHz}$ rate and then filtered to a working bandwidth of $30 \mathrm{kHz}$, the filtered output signal is adequately represented by a $60 \mathrm{kHz}$ sample rate. The decimating filter removes the unnecessary samples so that the subsequent processing stages in the system are presented with a more manageable, and meaningful, data rate.

The low-rate filtered data are then fed to the final processing block where they are given a time tag associated with an absolute GPS time reference. These time-tagged data are briefly stored and then transmitted in a message block to the spacecraft control and Data Handling 
Unit (DHU). A GPS time reference is received from the DHU every second. Between GPS updates the local clock providing the time stamp is required to be accurate to $\pm 8 \mu$ s.

The preamplifier gain, the LO characteristics, the filter characteristics and the decimation factor are all set by the Channel Control block. These settings are uploaded and changed on command from the spacecraft DHU. The Channel Control block also monitors the RRI internal status and built-in test (BIT) results, reporting this information to the DHU as telemetry.

The "Store and Forward, Time Tag" and "Channel Control" blocks are implemented in an Analog Devices ADSP-2191M Digital Signal Processor (DSP) under software control. This software is loaded on startup from the Local Software Storage block, an EEPROM. This software can be modified or upgraded by the spacecraft DHU. Hence, a software upload data path is shown. Also, the channel control block can check the integrity of the Software Storage and report integrity back to the DHU within the telemetry stream.

Finally, two support functions are shown at the bottom: the "Local Power Supplies" block, and the "Local Digital System Clock(s)". The Power Supplies block converts and regulates the voltages and provides the current flow required by the RRI electrical devices from the Spacecraft 28 VDC unregulated power source. It also provides the required electromagnetic interference filtering. The Digital Clock(s) provides the local clock signals (plural since more than one frequency and/or phase is needed), sourced from a stable crystal oscillator, required by the synchronous digital electronics.

Figure 6 shows the devices that were selected to perform the functions of the DRRM. It consists of two circuit cards each encapsulated within a compartment of a larger enclosure: the Digital Radio Receiver (DRR) circuit and the Power Supply (PS) circuit. Isolating the cards provides electromagnetic shielding from power supply noise. The DRR accepts the analog signals from each preamplifier over twisted shielded-pair cables. The first action performed is to digitize the signals with AD9238 dual 12-bit analog-to-digital converters. These devices provide numerous benefits: an onboard input differential amplifier to support the analog differential mode of operation, an output multiplexer to match the multiplexed digital inputs of the Digital Down Converter (DDC), and very low power consumption, approximately $700 \mathrm{~mW}$.

An AD6624 four-channel digital down converter is the heart of the digital receiver. It routes up to four input data streams to any, or all, of the four internal processing channels. The characteristics of each channel are individually programmable. A 32-bit numerically controlled oscillator mixes the input signals down to the baseband producing I and $\mathrm{Q}$ outputs. These are then immediately low-pass filtered and decimated with a Cascaded Integrator-Comb (CIC) filter. The CIC is followed by a 160-tap RAM coefficient (RCF) finite impulse response filter, also with the capability of further decimating its output to more efficiently match the data rate to the signal's new, reduced pass bandwidth. It is the CIC in combination with the RCF that are used to implement the 10 - and $30-\mathrm{kHz}$ output bandwidths specified for this instrument. Design of the filter coefficients to achieve the desired DDC response is performed using Analog Devices' SoftCell filter design tool. The four output data streams are then multiplexed together onto a single synchronous data link for hand-off to the digital signal processor (DSP).

An Analog Devices ADSP2191M fixed-point DSP is used as the RRI's central processor. Its primary function is to acquire data from the DDC, package it according to the experiment being performed, add a time stamp and then pass the resultant data packets to e-POP's Data Handling Unit over a synchronous, differential RS422 serial data link. However, it is also the RRI's primary command and telemetry interface processor. In advance of a specific experiment it programs the DDC characteristics and the AD5327 DAC with the appropriate preamplifier gain settings. This DSP was chosen both for its data processing capability and 


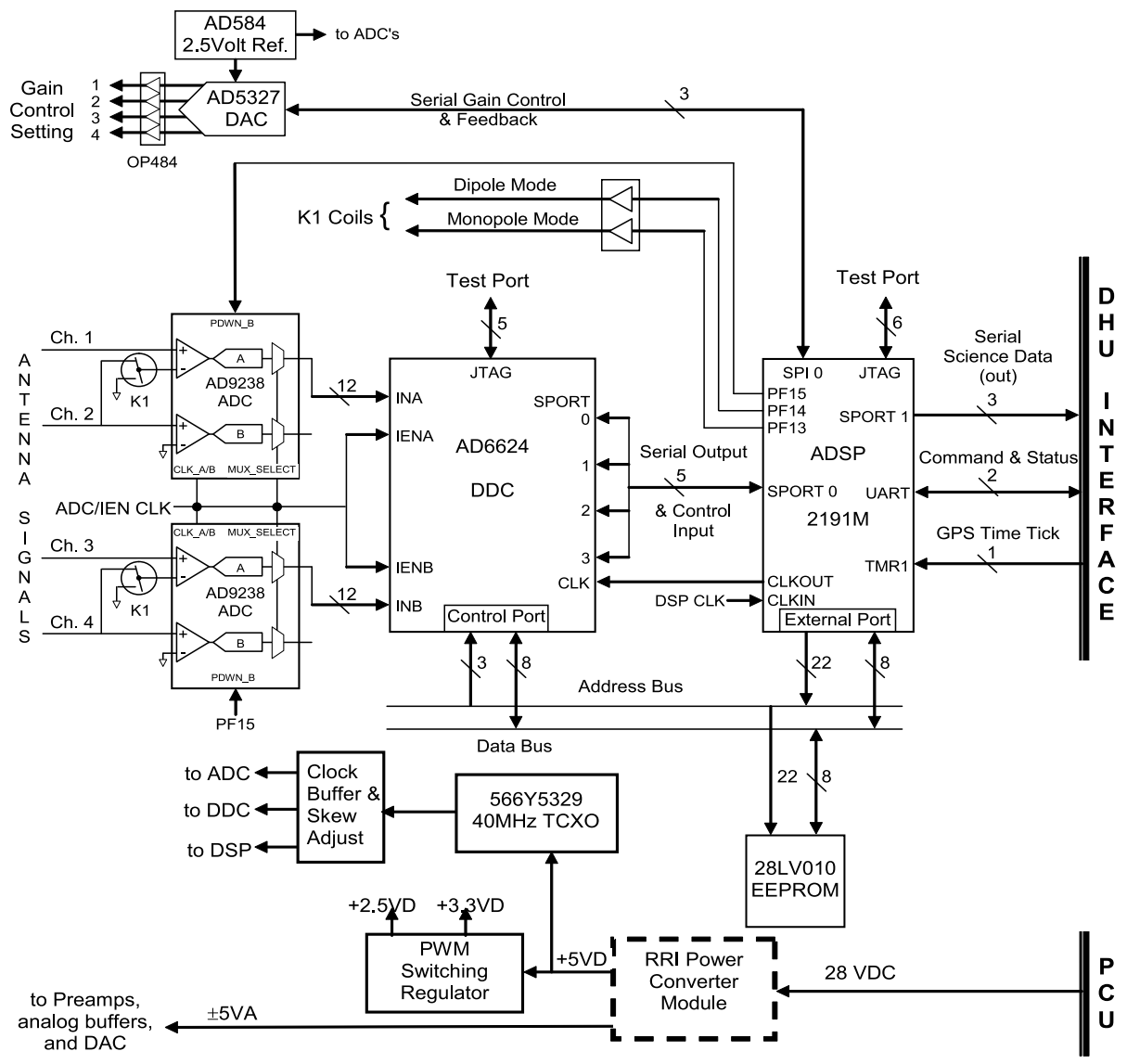

Fig. 6 Digital Radio Receiver Module block diagram

its rich variety of interface ports. DSP software is loaded on power-up from a Maxwell Technologies 28LV010, a 128k-by-8-bit radiation-tolerant EEPROM. In the event of EEPROM data corruption, the DSP can be booted-up directly from the DHU and the EEPROM can be reprogrammed.

Finally, the DRR clock signals are all synthesized from a Vectron International 566Y5329 40-MHz thermally controlled crystal oscillator.

\subsection{Tabular Summaries of RRI Design Objectives}

Table 1 is a list of the main parameter design goals for RRI operations. The characterization report in Sect. 3 will show that these objectives have been largely met. Table 2 summarizes the resource requirements of the RRI on CASSIOPE.

\subsection{Commercial Parts}

A notable aspect of RRI as a space instrument is its reliance on commercial-off-the-shelf (COTS) parts, as opposed to traditional space-qualified, radiation-hard parts, for its electronics. This design approach was largely a cost and power saving measure. Consequently, 
Table 1 Parameter ranges

\begin{tabular}{ll}
\hline Parameter & Value \\
\hline Preamplifier & \\
Input impedance & $>10^{11} /\left(\omega\left[\mathrm{s}^{-1}\right]\right) \Omega$ \\
Low level signal range-High gain & $0.32-1260 \mu \mathrm{V}$ \\
Mid level signal range-Medium gain & $0.010-39.8 \mathrm{mV}$ \\
High level signal range-Low gain & $0.32-1260 \mathrm{mV}$ \\
& \\
Digital radio receiver & \\
Frequency range & $10 \mathrm{~Hz}-18 \mathrm{MHz}$ \\
Baseband sampling rate & $62,500 \mathrm{~s}-1$ \\
Full scale sample range & $2^{15}$ \\
Pass bandwidths & $10,30 \mathrm{kHz}$ \\
Settling time & $\leq 100 \mu \mathrm{s}$ \\
Time stamp accuracy & $\pm 8 \mu \mathrm{s}$ \\
Output streams & Four $(\mathrm{I}$ or Q $)$ \\
Total data rate & $3.84 \mathrm{Mbits} \mathrm{s}-1$ \\
Environment & \\
Survival temperatures & \\
Operating temperatures & $-20^{\circ}$ to $60{ }^{\circ} \mathrm{C}$ \\
Tolerable radiation dose & $-10^{\circ}$ to $40^{\circ} \mathrm{Crad}$ \\
\hline
\end{tabular}

Table 2 Resource requirements of the RRI

\begin{tabular}{|c|c|c|c|c|}
\hline Component & Mass, $\mathrm{kg}$ & Volume, $\mathrm{cm}^{3}$ & Dimension, $\mathrm{cm}$ & Power, W \\
\hline \multicolumn{5}{|l|}{ Preamplifier } \\
\hline Monopole antenna + deployer (ea.) & 0.23 & - & $7.9 \times 8.3$ (dia. $)$ & - \\
\hline Deployer bracket (ea.) & 0.16 & 720 & $13 \times 8.3 \times 6.7$ & - \\
\hline Circuit Card Assembly (ea.) & 0.05 & - & $7.9 \times 7.9$ & 0.55 \\
\hline Enclosure and hardware & 3.16 & 2900 & $13 \times 13 \times 17$ & - \\
\hline Total & 4.92 & 5800 & $30 \times 30 \times 18$ & 2.2 \\
\hline \multicolumn{5}{|l|}{ Digital Radio Receiver Module } \\
\hline Enclosure and hardware & 1.52 & 1600 & $20 \times 16 \times 5$ & - \\
\hline Digital radio receiver circuit & 0.25 & - & $5.4 \times 3.1$ & 3.3 \\
\hline Power supply circuit & 0.22 & - & $5.1 \times 2.4$ & 2.0 \\
\hline Total & 1.99 & 1600 & $20 \times 16 \times 5$ & 5.3 \\
\hline \multicolumn{5}{|l|}{ Cable Harness } \\
\hline Connector and backshell (ea.) & 0.08 & & & \\
\hline Cable (2 m @ $\left.170 \mathrm{~g} \mathrm{~m}^{-1}\right)$ & 0.34 & & & \\
\hline Total & 1.68 & & & \\
\hline Instrument total & 8.68 & 7400 & - & 7.5 \\
\hline
\end{tabular}


Fig. 7 Radiation dose/depth prediction
CASSIOPE dose/depth (Solar Min)

$350-1500 \mathrm{~km} / 80$ degrees

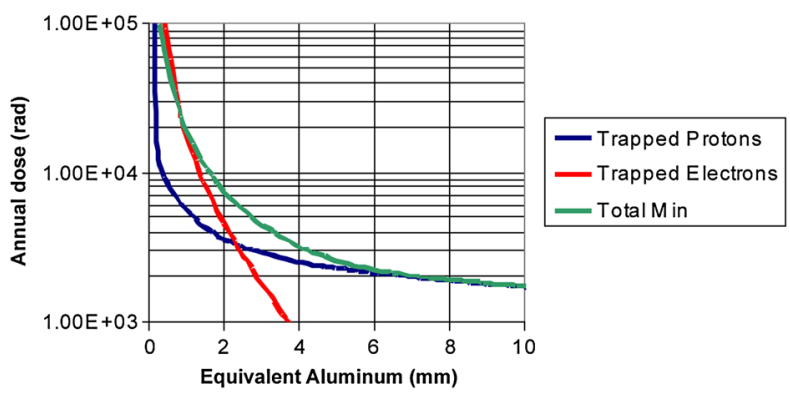

the radiation performance of the instrument is difficult to predict. Technology parameters that determine radiation hardness are not intentionally controlled during manufacture of COTS parts. As a result, the Total Ionizing Dose (TID) hardness of COTS technologies is often quite variable.

Because of the concern of the performance of these COTS parts in the space environment, a radiation analysis was performed by modeling the CASSIOPE spacecraft orbit. This analysis allowed us to determine the appropriate degree of shielding for the instrument. The results are shown in Fig. 7. From knowledge of the radiation performance of sub-micron MOS parts, it is believed that the annual dosage should be below $2 \mathrm{krad}$. Hence it was concluded that aluminum enclosure walls of approximately $7 \mathrm{~mm}$ should provide adequate shielding. Since the spacecraft structure contributes some shielding, it was decided to make the enclosures $5 \mathrm{~mm}$ thick.

\subsection{Custom Command Mode Parameters}

The command parameters used to completely specify the RRI operating configuration are listed with reference to the three major functional areas highlighted with three different colors in Fig. 2. With reference to the details of Fig. 6, channel numbers 1 through 4 connect to the monopoles with those numbers. On the same Figure, for dipole antenna connection, the A settings are the channel-1 settings because that is the signal that is processed for the 1-2 dipole signal and routed to DDC input A. Similarly the B settings are the channel-3 settings, which put out the 3-4 dipole signal routed to DDC input B. In the case of monopole signal processing, the AD9238 output multiplexers route all four monopole signals to the DDC A and B inputs. The parameter Data Format selects which four of I1 through Q4 streams are to be recorded. Here is a complete list of the RRI Custom Command Mode parameters:

- Preamplifier (pink)

GAIN 1: Low, medium, high (for channel 1)

GAIN 2: Low, medium, high (for channel 2)

GAIN 3: Low, medium, high (for channel 3)

GAIN 4: Low, medium, high (for channel 4)

- Monopoles Antenna (yellow)

SWITCH K1: monopole, dipole

- Analog-Digital Converter and Digital Down Converter (blue) 
SWEEP A: Fixed frequency, Linear sweep, or Logarithmic Sweep, for input A.

BANDWIDTH A: $30 \mathrm{kHz}$ or $10 \mathrm{kHz}$, for input A.

F1A: The channel 1 frequency setting in fixed frequency, or the input-A initial frequency setting in a sweep.

F2A: The channel 2 frequency setting in fixed frequency, or the input-A terminal frequency setting in a sweep.

$\triangle F A$ : In fixed frequency, zero.

In linear sweep, this sets the frequency step size of input A.

In logarithmic sweep, this sets the initial frequency step size for input A. Subsequent steps are twice the previous step.

Dwell A: In fixed frequency, zero.

In sweep modes, the length of time spent at each step. Value is an integer multiple of the time of four science data packets $(0.001856 \mathrm{~s})$.

SWEEP B: Fixed frequency, Linear sweep, or Logarithmic Sweep, for input B.

BANDWIDTH B: $30 \mathrm{kHz}$ or $10 \mathrm{kHz}$, for input B.

F1B: The channel 1 frequency setting in fixed frequency, or the input-B initial frequency setting in a sweep.

F2B: The channel 2 frequency setting in fixed frequency, or the input-B terminal frequency setting in a sweep.

$\triangle F A$ : In fixed frequency, zero.

In linear sweep, this sets the frequency step size of input B.

In logarithmic sweep, this sets the initial frequency step size for input B. Subsequent steps are twice the previous step.

Dwell B: In fixed frequency, zero.

In sweep modes, the length of time spent at each step. Value is an integer multiple of the time of four science data packets $=0.001856 \mathrm{~s}$.

Data Format: Designates which four of eight possible data streams are recorded. This is a 2-byte number. E.g., for I1-Q1-I3-Q3, the two-byte word is 0x0145.

Further information about commands can be found in the RRI User's Manual (U. Calgary 2008a). The RRI command scheme was first envisaged for twelve instrument modes, each with a default assignment of all the parameters. During the characterization of the RRI, it was judged expedient to simplify RRI commands in terms of the instrument parameters, i.e., to adopt the Custom Command approach. This lets the e-POP mission operation provide practical experience as to which RRI modes should be inventoried, together with their nicknames and Custom Command parameter assignments.

We conclude Sect. 2 with reference to the photograph in Fig. 8 of the finished RRI hardware. On the left are the antenna deployer units integrated with the preamplifier enclosure unit. These are installed on the external $x$-face of the spacecraft as illustrated in Fig. 1. On the right is the DRRM, which is housed in the interior of the spacecraft.

\section{Characterization}

\subsection{Preamplifier Input Impedance and Antenna Mismatch}

A design goal of the RRI was to be able to operate the monopole antennas as voltage probes. When the preamplifier input impedance $Z_{o}$ is much greater than the monopole impedance $Z_{\text {ant }}$, the measured input voltage $V_{i n}$, is the same as the open-circuit monopole voltage $V_{o c}$. 
Fig. 8 Photograph of the RRI hardware. The external part on the left is comprised of the monopole deployers in white and the central preamplifier enclosure box. The DRRM on the right is housed in the internal space of the spacecraft
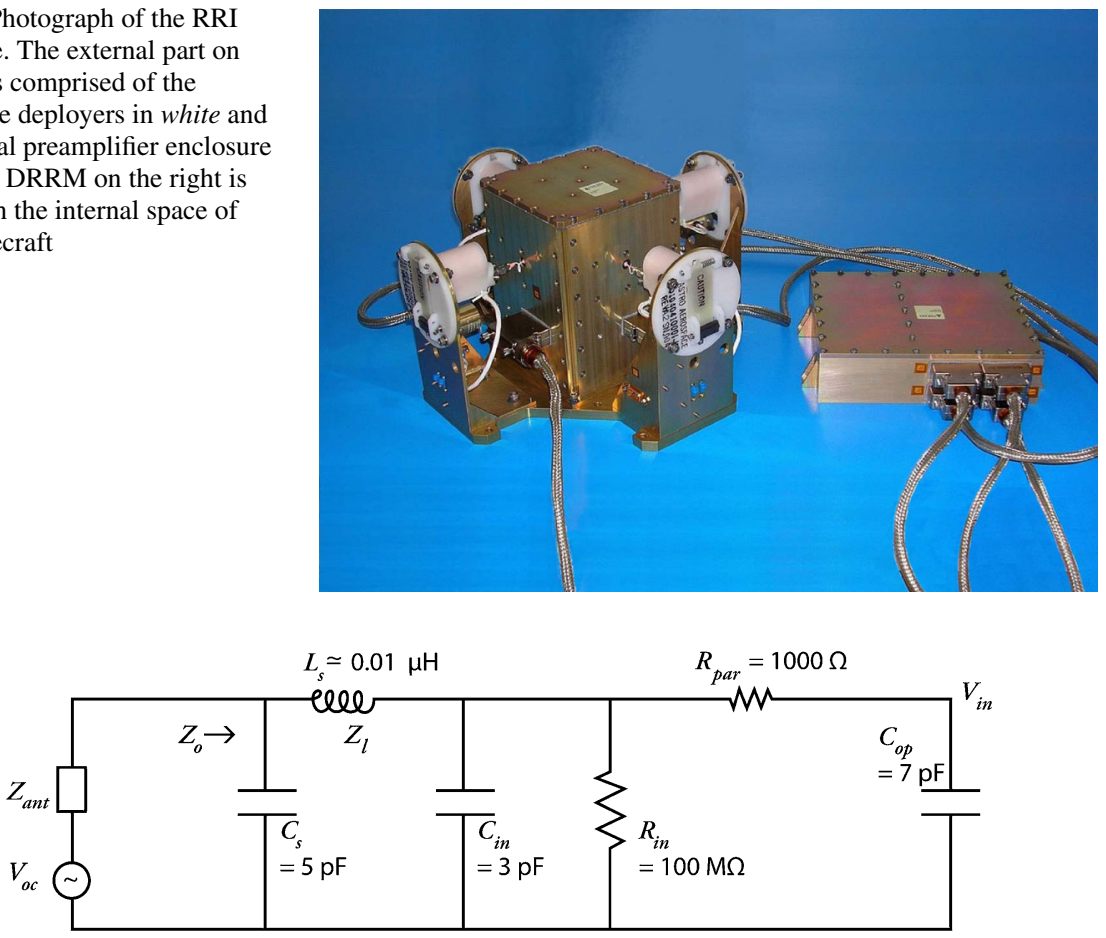

Fig. 9 Equivalent circuit of the monopole antenna and the PA input

Applying some hypothesis about the monopole effective length $L_{\text {eff }}$, the input voltage measurement leads directly to the magnitude of the wave electric field in the medium $E_{z}$ through the relation $E_{z}=V_{o c} / L_{e f f}$, subscript $z$ indicating the direction of the monopole.

The principal elements of the input equivalent circuit are depicted in Fig. 9. $C_{s}$ is the measured stray capacitance between the deployed STEM antenna and ground, $C_{i n}, R_{i n}$, and $R_{p a r}$ are the essential parts of the PA circuit hardware and $C_{o p}$ is the capacitance of the first operational amplifier in the preamplifier.

Swept- and fixed-frequency bridge methods were applied at different frequencies to the determination of $Z_{o}$. It was concluded that the effective input equivalent circuit for PAs in the engineering model PA enclosure is the parallel combination of a $100-\mathrm{M} \Omega$ resistance and a 12-pF capacitance.

When the measured input impedance is inserted into the equivalent circuit for the PA front end, the result is the mismatch shown in Fig. 10. Here the ratio of the open-circuit antenna-terminal voltage to the Op-Amp front-end voltage for dipole orientations parallel $\left(0^{\circ}\right)$ and perpendicular $\left(90^{\circ}\right)$ to the earth's magnetic field is plotted as a function of frequency, for e-POP apogee and perigee condition examples. These calculations employ the Balmain (1969) cold-plasma dipole theory for $f<3 \mathrm{MHz}$, and vacuum dipole theory for $f>3 \mathrm{MHz}$. For our assumed perigee conditions $\left(f_{p e}=3.1 \mathrm{MHz}, f_{c e}=1.3 \mathrm{MHz}\right.$, electronneutral collision frequency $=1 \mathrm{kHz}$ ), the theory predicts a singular dipole impedance close to the upper hybrid resonance frequency at 3.4 MHz. The sharp swings of the ratio in Fig. 10 serve as a reminder of the care that must exercised in interpreting spontaneous emissions in this parameter neighborhood. Otherwise, smoothly maintained ratio values near 1 indicate that mismatch is not a big problem for most other frequencies below $18 \mathrm{MHz}$. 
Fig. 10 Ratio of the monopole open-circuit voltage to the input voltage, calculated for two different ionospheric conditions, and for two different angles between the monopole axis and the Earth's magnetic field

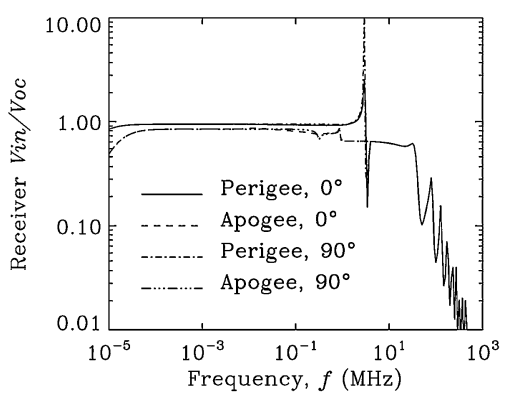

\subsection{Pass Band Response}

The end-to-end complex pass band response $H(f)$ of the RRI has been characterized as the product of three functions:

$$
H(f)=R\left(f_{B B}\right) h(f) \exp [\mathrm{i} \Phi(f)] .
$$

In expression (1),

$f \quad$ is the frequency offset from pass band center;

$R\left(f_{B B}\right)$ is the absolute dynamic response factor. It is the equivalent of gain but essentially a ratio, because its units are digital numbers out, divided by analog peak-peak voltage at the input;

$f_{B B} \quad$ is the broadband frequency of pass band center, where $10 \mathrm{~Hz}<f_{B B}<18 \mathrm{MHz}$. It is set with the 4-byte number NCO;

$h(f)$ is the relative amplitude pass band response of the receiver;

$\Phi(f) \quad$ the pass band phase response.

Sections 3.2.1, 3.2.2, and 3.2.3 report on the three functions in Eq. (1) while $f_{B B}$ is kept constant at 7.4, 18, 21 or $10000 \mathrm{kHz}$. Then, in Sect. 3.2.4, the broadband dependence of $R$ on $f_{B B}$ is set forth.

\subsubsection{Absolute Dynamic Response Factor, $R$}

Factor $R$ is probably the most frequently applied calibration of the RRI and was measured at pass band centre. Twelve values of $R$ were tabulated in the linear part of the dynamic response, that is, one for each of three gain values and for each of the four preamplifiers.

$R$ values were characterized for monopoles 1 and 2, with ganged switch K1 in Fig. 2 in a position to allow those monopole I and Q signals to be recorded separately. This is called the monopole mode. Channels 3 and 4 were similarly tested with the switch K1 similarly in the grounded position. A typical plot of output digital level versus input analog peak-peak voltage is presented in Fig. 11, for monopoles 3 and 4 with PA gain in the "High" setting. The straight red-line fit to the linear part of the curve corresponds to a $R$ value of 3.47 digital numbers per microvolt input to channel 3 . The straight-line fits usually span at least $70 \mathrm{~dB}$ of linear response in input voltage. With the exception of noise threshold at the lower left, there is negligible disparity between channels 3 and 4 .

The results of straight-line fits to the linear parts of all of the aforementioned $12 \mathrm{com}$ binations of monopoles, for $f_{B B}=21$ and $10000 \mathrm{kHz}$, and for the three PA gain states are gathered into Tables 3 and 4. For completeness, just above the $R$ values, the 2-byte hexadecimal command codes sent from the RRI Digital Signal Processor to the preamplifiers are shown. 
Fig. 11 An example of an amplitude dynamic response curve, for channels 3 and 4, SWITCH K1 selecting monopole, and GAIN High. The red-line fit is the dynamic response $R=3.47$ digital numbers per microvolt peak-peak input

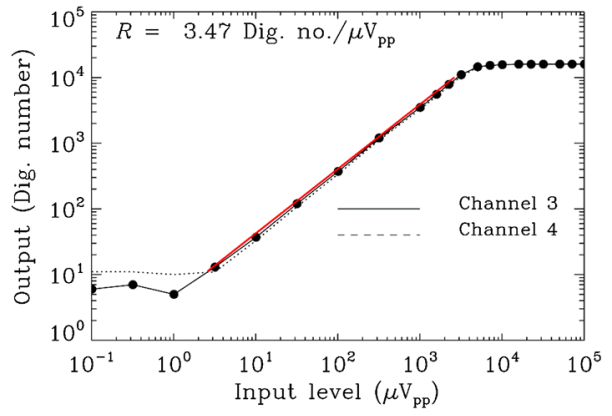

Table 3 Summary of monopole dynamic response factors $R$ for $f_{B B}=21 \mathrm{kHz}$

\begin{tabular}{|c|c|c|c|c|c|}
\hline \multirow[t]{2}{*}{ Gain name } & & \multicolumn{4}{|c|}{ Channel No. } \\
\hline & & 1 & 2 & 3 & 4 \\
\hline \multirow[t]{2}{*}{ Low } & Command & $02 \mathrm{CD}$ & 4263 & $82 \mathrm{~A} 8$ & C2EB \\
\hline & $R$ (Dig. Nos. $\left./ \mu \mathrm{V}_{\mathrm{pp}}\right)$ & 0.00337 & 0.00337 & 0.00360 & 0.00360 \\
\hline \multirow[t]{2}{*}{ Medium } & Command & $08 \mathrm{C} 7$ & 485D & 88A1 & $\mathrm{C} 8 \mathrm{EC}$ \\
\hline & $R$ (Dig. Nos. $\left./ \mu \mathrm{V}_{\mathrm{pp}}\right)$ & 0.107 & 0.107 & 0.114 & 0.114 \\
\hline \multirow[t]{2}{*}{ High } & Command & 0EC7 & $4 \mathrm{E} 5 \mathrm{D}$ & $8 \mathrm{~EB} 4$ & CEF9 \\
\hline & $R$ (Dig. Nos. $/ \mu \mathrm{V}_{\mathrm{pp}}$ ) & 3.09 & 3.09 & 3.47 & 3.47 \\
\hline
\end{tabular}

Table 4 Summary of monopole dynamic response factors $R$ for $f_{B B}=10000 \mathrm{kHz}$

\begin{tabular}{|c|c|c|c|c|c|}
\hline \multirow[t]{2}{*}{ Gain name } & & \multicolumn{4}{|c|}{ Channel No. } \\
\hline & & 1 & 2 & 3 & 4 \\
\hline \multirow[t]{2}{*}{ Low } & Command & $02 \mathrm{CD}$ & 4263 & $82 \mathrm{~A} 8$ & $\mathrm{C} 2 \mathrm{~EB}$ \\
\hline & $R$ (Dig. Nos. $\left./ \mu \mathrm{V}_{\mathrm{pp}}\right)$ & 0.00225 & 0.00225 & 0.00237 & 0.00237 \\
\hline \multirow[t]{2}{*}{ Medium } & Command & $08 \mathrm{C} 7$ & $485 \mathrm{D}$ & $88 \mathrm{~A} 1$ & C8EC \\
\hline & $R$ (Dig. Nos. $\left./ \mu \mathrm{V}_{\mathrm{pp}}\right)$ & 0.0680 & 0.0680 & 0.0710 & 0.0710 \\
\hline \multirow[t]{2}{*}{ High } & Command & 0EC7 & 4E5D & 8EB4 & CEF9 \\
\hline & $R$ (Dig. Nos. $\left./ \mu \mathrm{V}_{\mathrm{pp}}\right)$ & 1.89 & 1.89 & 2.08 & 2.08 \\
\hline
\end{tabular}

In like manner to monopole $R$ characterization, the $R$ values for dipoles 1,2 (the dipole formed with monopoles 1 and 2 by closing switch K1) and 3, 4 were obtained. Twelve values of $R$, that is, for each of three gain values, for each of two dipole inputs and for each of the two frequencies have been tabulated in Table 5. In Fig. 6, the ganged switch K1 was set to combine the channel-1 and -2 signals differentially in the upper AD9238 analog-digital converter, and the AD6624 put out the resulting I1 and Q1 digital waveforms. Similarly, the switch $\mathrm{K} 1$ position allowed the I3 and Q3 signals to be recorded from the 3, 4 dipole.

The individual plots of output versus input together show that the linear response $R$ values can be applied in routine processing for output values between 20 and 10000 digital numbers. Hands-on special attention is required for analysis of output numbers outside that range. 
Table 5 Summary of dipole dynamic response factors $R$ for $f_{B B}=21 \mathrm{kHz}$ and $10000 \mathrm{kHz}$

\begin{tabular}{|c|c|c|c|c|c|}
\hline \multirow[t]{3}{*}{ Gain name } & \multirow[t]{3}{*}{ Dipole No. } & \multicolumn{4}{|l|}{$f_{B B}$} \\
\hline & & \multicolumn{2}{|l|}{$21 \mathrm{kHz}$} & \multicolumn{2}{|l|}{$10000 \mathrm{kHz}$} \\
\hline & & 1,2 & 3,4 & 1,2 & 3,4 \\
\hline \multirow[t]{2}{*}{ Low } & Command & $02 \mathrm{CD}, 4263$ & $82 \mathrm{~A} 8, \mathrm{C} 2 \mathrm{~EB}$ & $02 \mathrm{CD}, 4263$ & 82A8, C2EB \\
\hline & $R$ (Dig. Nos. $\left./ \mu \mathrm{V}_{\mathrm{pp}}\right)$ & 0.00312 & 0.00324 & 0.00213 & 0.00223 \\
\hline \multirow[t]{2}{*}{ Medium } & Command & 08C7, 485D & 88A1, C8EC & $08 \mathrm{C} 7,485 \mathrm{D}$ & 88A1, C8EC \\
\hline & $R$ (Dig. Nos. $\left./ \mu \mathrm{V}_{\mathrm{pp}}\right)$ & 0.0968 & 0.0977 & 0.0639 & 0.0616 \\
\hline \multirow[t]{2}{*}{ High } & Command & 0EC7, 4E5D & 8EB4, CEF9 & 0EC7, 4E5D & 8EB4, CEF9 \\
\hline & $R$ (Dig. Nos. $\left./ \mu \mathrm{V}_{\mathrm{pp}}\right)$ & 2.89 & 3.02 & 1.78 & 1.95 \\
\hline
\end{tabular}

Fig. 12 The shape of the $30-\mathrm{kHz}$ pass band relative amplitude response $h(f)$, including the custom mode parameters used to obtain the data. Dots represent the measurements, which are joined by straight lines

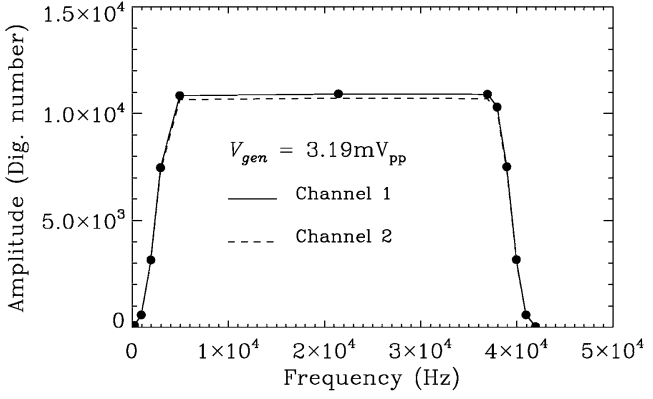

RRI Custom Mode parameters

$\begin{array}{lll}\text { SWITCH K1 Monopole } & \text { SWEEP A Fixed Frequency } & \text { SWEEP B Fixed Frequency } \\ \text { GAIN1 High } & \text { BANDWIDTH A } 30 \mathrm{kHz} & \text { BANDWIDTH B } 30 \mathrm{kHz} \\ \text { GAIN2 High } & \text { F1A 21 kHz } & \text { F1B 21 kHz } \\ \text { GAIN3 High } & \text { F2A 21 kHz } & \text { F2B 21 kHz } \\ \text { GAIN4 High } & \triangle \text { FA NA kHz } & \text { DFB NA kHz } \\ & \text { DWell A NA ms } & \text { Dwell B NA ms } \\ & & \text { Data Format I1Q112Q2 }\end{array}$

Reviewing Tables 3 through 5 qualitatively, one sees that PA gain hex commands have been successfully adjusted to give the same, or at least very similar, $R$ values in the two opposing monopoles that make up a dipole. Nevertheless, comparing a monopole table cell with its corresponding cell in the dipole table, the monopole $R$ value is slightly higher than that of the dipole. Comparing the two monopole tables, cell for cell, $R$ values are higher at $21 \mathrm{kHz}$ than at $10000 \mathrm{kHz}$, demonstrating the amplification roll-off in the RRI broadband response. More detail about the broadband response is documented in Sect. 3.2.4 below.

\subsubsection{Relative Amplitude Response, $h$}

Function $h(f)$ in Eq. (1) was measured at as many $f$ values as was needed to establish the entire bandwidth amplitude response down to noise threshold on both sides of band center. The RRI uses two pass bandwidth (BW) values, nominally 10 and $30 \mathrm{kHz}$. The response was recorded for both BWs, for all four monopoles and both dipoles, and for all three levels of PA gain. These characterizations were carried out using generator levels that kept digital output at maximum values in the linear part of the dynamic response, at about 11000.

The shapes of $h(f)$ are constant for a given BW value, independent of the antenna and PA gain configuration. The $30-\mathrm{kHz}$ shape is represented by Fig. 12, which has not been normalized to unity at band center. In this plot of digital output versus frequency, measured 
Fig. 13 The lowest-frequency part of the non-normalized $h(f)$ in Fig. 12, with the same parameters except $\mathrm{F} 1 \mathrm{~A}=\mathrm{F} 2 \mathrm{~A}=$ $18 \mathrm{kHz}$

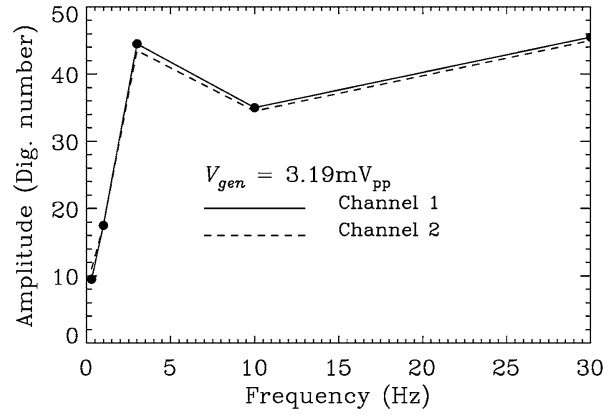

Fig. 14 The 10-kHz pass band non-normalized relative amplitude response $h(f)$

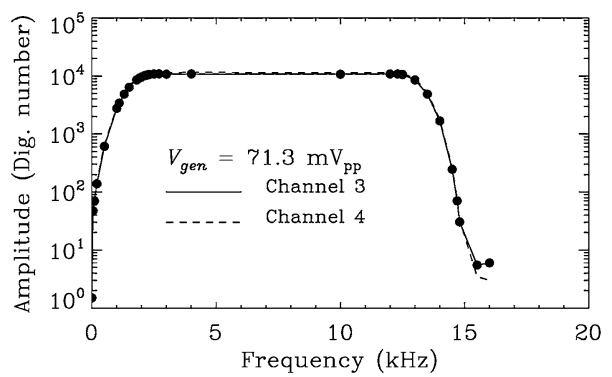

points in Channel 1 are the dots. At the bottom is the set of Custom Mode parameters that completely specifies the configuration of the RRI. The $V_{\text {gen }}$ value displayed is the input voltage to channel 1 and to channel 2.

Figure 12 reveals the expected, desired shape of the RRI pass band. It exhibits a flat response out to BW limits, outside of which there is steep roll-off. The digital down converter of the RRI is not operated in baseband mode. All frequencies down to about $1 \mathrm{~Hz}$ can be covered using nonzero band-center frequencies NCO; these particular values of NCO had to be found by careful adjustment during testing.

The non-normalized relative response at the lowest possible frequencies is illustrated in Fig. 13. Here, the same parameters as in Fig. 12 are used except that the required NCO value-both "F1A" and "F2A"- - needed to be set to $18 \mathrm{kHz}$. In this $30-\mathrm{kHz}$ nominal BW case, a F1A setting of $18 \mathrm{kHz}$ covers all frequencies down to $0.3 \mathrm{~Hz}$ while avoiding negative frequencies that are folded back into the positive values, which would confuse the frequency analysis. A measurable level of about 10 is obtained at $1 \mathrm{~Hz}$, suggesting that with judicious choice of PA gain setting, it may be possible to measure electric fields below the nominal lower frequency limit of the RRI at $10 \mathrm{~Hz}$. The local peak in the response at $3 \mathrm{~Hz}$ was reproducible, but it remained unexplained.

Figure 14 shows the non-normalized relative $10-\mathrm{kHz}$ response $h(f)$, now with channels 3 and 4 being exercised. In this case, F2A and F2B needed to be set to $7.4 \mathrm{kHz}$ to measure in the total available frequency range.

The pass band amplitude response was documented also for F1A, F1B, F2A, F2B = $10 \mathrm{MHz}$. The same response shapes $h(f)$ were obtained for the two BW as in Figs. 12 and 14.

The relative pass band response is uniquely determined by the digital signal processing and filtering in the digital down converter (blue) part of the RRI in Fig. 2. Therefore the responses just reported for the monopole excitation apply equally to dipole excitation. 
Fig. 15 The Broad Band non-normalized amplitude response

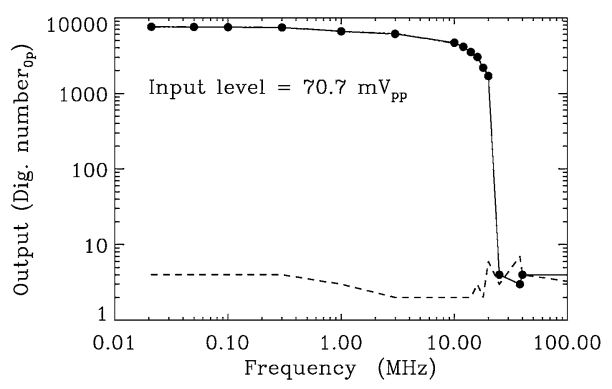

\subsubsection{Relative Phase Response, $\Phi(f)$}

The phase response function with respect to frequency offset, $\Phi(f)$, in Eq. (1) was achieved using a finite-impulse-response (FIR) filter in the DDC. The design objective of linearity of the RRI phase with $f$ provides constant delay through the filter at all frequencies, avoiding signal distortion. The FIR filter in the RRI has $N=160$ taps, and the DDC sampling frequency is $f_{s}=62500 \mathrm{~s}^{-1}$. Assuming that the FIR filter is the sole determinant of phase response, the delay should be $d=(N-1) / f_{s}=2.544 \mathrm{~ms}$, and the relative phase delay across the bandwidth should be:

$$
\Phi_{F I R}=-d f
$$

During characterization, (2) was examined by applying a linearly-swept-frequency signal to both dipole inputs of the RRI. From the phase $\Phi=\arctan (\mathrm{Q} / \mathrm{I})$ of each pair of in-phase (I) and quadrature $(\mathrm{Q})$ values, the instantaneous frequency of the sweep could be found by multiplying the difference of consecutive $\Phi$ values by $f_{s} / 2 \pi$.

These measurements confirmed the linear phase response by showing that both the phase and the frequency $f_{m}$ measured as a function of time across a FM sweep agree with what (2) implies. It was found for both $f_{m}$ and $\Phi$ that their deviation from pure linear responses over realistic data-recording times $(\leq 1 \mathrm{~s})$ is not detectable in the RRI output. It was concluded that the right side of the linear phase relation (2) can be inserted for $\Phi(f)$ into (1) with $d=2.544 \mathrm{~ms}$.

\subsubsection{Broadband Amplitude Response}

The amplification factors $R\left(f_{B B}\right)$ appearing in Eq. (1) were found to vary by up to several times across the RRI broad bandwidth, $10 \mathrm{~Hz}<f_{B B}<18 \mathrm{MHz}$. To determine $R$ values at some general frequency, the broadband amplitude response as a function of frequency was explored, using now a series of band-center frequencies F1A, F1B, F2A, and F2B spanning the range $10 \mathrm{~Hz}$ to $18 \mathrm{MHz}$.

The resulting Fig. 15 shows how output level varies with frequency across the broad band when the input level is held constant. A monopole voltage of $70.7 \mathrm{mV}_{\mathrm{pp}}$ and a "Medium" gain setting were used, and the I2 and I3 channels are plotted. The overall shape of the response owes, in first approximation, to the frequency characteristics of the PAs, while the sharp fall off at $18 \mathrm{MHz}$ results from the 5-pole Butterworth anti-aliasing filters inserted between the PAs and the ADCs. The values of $R$ reported for $21 \mathrm{kHz}$ and $10000 \mathrm{kHz}$ at medium gain in Tables 3 and 4 are recovered here.

Expressions of digital number out as quadratic functions of frequency were found to fit the curves closely when the total band is divided into two sub bands: 
Table 6 Aliasing rejection

\begin{tabular}{rrrr}
\hline NCO & \multicolumn{1}{l}{$f_{\text {gen }}$} & $V_{\text {gen }}$ & Output (Dig. No.) \\
\hline $5 \mathrm{MHz}$ & $25 \mathrm{MHz}$ & $70.7 \mathrm{mV}_{0 \mathrm{p}}$ & 144 \\
$10 \mathrm{MHz}$ & $30 \mathrm{MHz}$ & $70.7 \mathrm{mV}_{0 \mathrm{p}}$ & 35 \\
$10 \mathrm{MHz}$ & $50 \mathrm{MHz}$ & $70.7 \mathrm{mV}_{0 \mathrm{p}}$ & 2 \\
$10 \mathrm{MHz}$ & $150 \mathrm{MHz}$ & $70.7 \mathrm{mV}_{0 \mathrm{p}}$ & 1 \\
\hline
\end{tabular}

Fig. 16 The measurementtheory disparity in phase shift increases with frequency

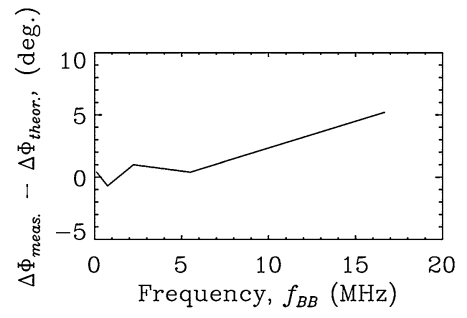

Dig. Output $=7571.87-256.17 f_{B B}-706.77 f_{B B}^{2} \quad$ for $0.018<f_{B B}<1.0 \mathrm{MHz}$, (3) or

$$
\text { Dig. Output }=6758.77-177.47 f_{B B}-3.7763 f_{B B}^{2} \quad \text { for } 1.0<f_{B B}<20.0 \mathrm{MHz} \text {. }
$$

In fact, the analytical curves, overlaid piecewise in Fig. 15, fit so well that they are hardly seen. These Dig. Output $\left(f_{B B}\right)$ curves are to be applied for all 3 gain settings. So, to determine $R$ for a general frequency $f_{B B}$ and gain setting, Dig. Output at $f_{B B}$ is evaluated using Eq. (3) or (4) and then divided by the test input voltage of $70.7 \mathrm{mV}_{\mathrm{pp}}$ to yield $R_{f}$. Apply (3) or (4) depending on whether $f_{B B}$ is above or below $1 \mathrm{MHz}$. Then, the value of $R$ is read from the "Medium" row in Tables 3, 4 or 5, taking again $1 \mathrm{MHz}$ as the dividing line between the $21 \mathrm{kHz}$ and $10 \mathrm{MHz}$ reference frequencies. The correction to be applied to $R$ anywhere in those tables is then $R_{f} / R$.

Our foregoing method for broadband frequency correction is illustrated in Fig. 15 for monopole- 2 and -3 signals, but is valid for all 4 monopoles and 2 dipoles.

\subsection{Anti-Aliasing}

It is clear from Fig. 15 that the design achieves more than $60 \mathrm{~dB}$ of anti-aliasing isolation of the $0-20 \mathrm{MHz}$ band from interference above $20 \mathrm{MHz}$. Four data files were recorded during characterization to document alias rejection. The digital number output levels in Table 6, arising at the aliased inband frequencies 5 and $10 \mathrm{MHz}$, are found to be very low, indicating the required rejection. The worst case in Table 6 of Output $=144$ corresponds to an input voltage of $2.1 \mathrm{mV}_{\mathrm{pp}}$ based on the appropriate factor $R=0.07$ in Table 4 .

\subsection{Antenna Phase Difference}

It is required to measure the amplitude and phase of signals induced on the RRI antennas to determine the electric polarization of wave fields incident on the spacecraft. Although the dipole antenna configuration may seem preferable for polarization measurements with its spatial symmetry, both monopoles and dipoles were examined. Amplitude response has already been dealt with in Sects. 1 and 2. Now the relative phase calibration is discussed. 
Table 7 Theoretical and measured phase differences

\begin{tabular}{llcl}
\hline$f_{B B}, \mathrm{MHz}$ & $L, \mathrm{~m}$ & \multicolumn{3}{c}{$\Delta \Phi,^{\circ}$} \\
\cline { 3 - 4 } & & Theor. & Meas. \\
\hline 0.100 & 22.4 & 4.07 & $4.5 \pm 0.1$ \\
0.744 & 22.4 & 30.3 & $29.6 \pm 0.1$ \\
2.232 & 22.4 & 90.9 & $91.9 \pm 0.1$ \\
5.500 & 3.00 & 30.3 & $30.7 \pm 0.2$ \\
5.500 & 3.66 & 36.6 & $39.5 \pm 0.5$ \\
8.333 & 2.44 & 37.0 & 41 \\
8.333 & 0 & 0 & $2.5,2.0$ \\
10.000 & 2.44 & 52.3 & 57 \\
16.6666 & 2.44 & 87.2 & 108 \\
16.6666 & 3.00 & 90.9 & $96.1 \pm 0.5$ \\
16.6666 & 3.66 & 111.0 & 121 \\
16.6666 & 0 & 0 & 12 \\
17.000 & 2.44 & 88.9 & 111 \\
\hline
\end{tabular}

The output of a fixed-frequency signal source was split twice with $\mathrm{T}$ junctions to replicate dipole signal components required for this analysis. The output from one side of the first $\mathrm{T}$ junction was fed through a length $L$ of RG58 cable to delay its phase with respect to the signal exiting from the other side of the T. Both signals were split with Ts a second time to provided inputs to the four monopole positions 1 through 4 in Fig. 6, with ganged switch K1 set to produce analogue dipole signals in channels 1 and 3 . The phase difference between the two complex wave forms $\mathrm{I} 1+\mathrm{jQ} 1$ and $\mathrm{I} 3+\mathrm{jQ} 3$ was computed and plotted from the measured difference in phase

$$
\Delta \Phi_{m}=\arctan (\mathrm{Q} 3 / \mathrm{I} 3)-\arctan (\mathrm{Q} 1 / \mathrm{I} 1) .
$$

The theoretical phase difference injected into the 1,2 arm by the delay line with a propagation speed of $0.66 c$ for waves at frequency $f_{B B}$ is

$$
\Delta \Phi_{t h}=2 \pi f_{B B} L / 0.66 c=1.819 f_{B B}(\mathrm{MHz}) L(\mathrm{~m})^{\circ} .
$$

A number of phase-difference theoretical values and measured phase differences are gathered in Table 7. These include both monopole and dipole cases. Figure 16 is restricted to the dipole measurements only.

In both Table 7 and Fig. 16, the disparity between observation and theory is seen to increase with frequency. This dependence may arise from an imbalance of the preamplifiers response functions. It is also possible that an internal timing characteristic of the RRI is the root cause of the disparity. For the present, the plan is to correct the dipole phase difference using a straight-line fit to Fig. 16. The plus-minus limits in the fourth column of Table 7 are the observed fluctuation in the measured phase difference over the measurement interval, typically $10 \mathrm{~ms}$.

If and when relative phase measurements to a few degrees are required, the analyst may be advised to apply a better fit to the data in Fig. 16, for instance, a ramp-up that breaks around $5 \mathrm{MHz}$. 


\section{Data Format and Timing}

\subsection{Data Format}

The e-POP Radio Receiver Instrument User's Manual (U. Calgary 2008a) and the e-POP Level 0 Data Description (U. Calgary 2008b) specify all command and telemetry formats required for this instrument. In orbit, an e-POP experiment lasts a period of time at the first of which all instrument parameters are set. For the rest of the experiment, the payload continues to operate with no change in those settings. In the case of the RRI experiments, analysts using its Level-Zero (.lv0) output data files are most interested probably in the "Sync Packet" and the "Custom Mode Packet".

RRI lv0 files, like files from the other e-POP instruments, follow a naming convention that specifies the instrument, time of recording and data quality (U. Calgary 2008b). At the beginning of a RRI file are four Sync Packets of length 272 bytes. The first 16 bytes of a Sync packet contain the Consultative Committee for Space Data Systems (CCSDS) recommended header for space data files (U. Calgary 2008b). The last 256 bytes include standard packet header information, instrument identifier, packet length, date and time to a precision of $12.5 \mathrm{~ns}, 116$ two-byte words of RRI parameter settings, and finally, two CRC bytes (U. Calgary 2008a). The parameter list in Sect. 2.7 above has the sequence in which the parameters appear in the RRI Sync packet.

The 272-byte Custom Mode Packet has the same assignments as the Sync Packet except that the aforementioned 116 words now contain the RRI digital output numbers. These are associated with the DDC "SPORT" outputs 0 through 3 in Fig. 6 and transmitted in a sequence repeated 29 times. For instance, in the case of Fig. 12, in each packet there were 29 repetitions of the data stream sequence I1 Q1 I2 Q2.

\subsection{Data Absolute Time}

An important operational objective of the RRI instrument is to determine absolute time of RRI data samples to $\pm 8 \mu \mathrm{s}$. In the design of e-POP, precise time is determined by combining information from two functions. First, GPS time is supplied to the nearest second by the e-POP GAP instrument. Second, the $80-\mathrm{MHz}$ oscillator of the RRI supplies ticks to a microsecond counter in the Signal Processing System. The 1-pps GPS signal causes the second counter to be reset once every four RRI packets. The microsecond counter is reset in the packet following the packet that was being transmitted when the 1-PPS signal arrived. Because the PPS can arrive at any time during the packet transmission cycle, the PPS interrupt priority may prevent immediate counter reset.

Thus, some care is required in correctly interpolating absolute time of RRI samples to microseconds. There is jitter in the determination of the true time in the general case when the 1-PPS arrives sometime during the 4 packets corresponding to a single 1-second count value. To avoid this jitter, software locates instances near the beginning of file when the 1-PPS signal arrives at the discrete break between one 4-packet group and the next. The microsecond counter has a small value, and the 1-PPS signal is then said to be handled in real time. Assuming that the RRI is a stable state machine, successive instances of real time in the data stream then allow accurate times to be interpolated, or extrapolated, throughout the file. 

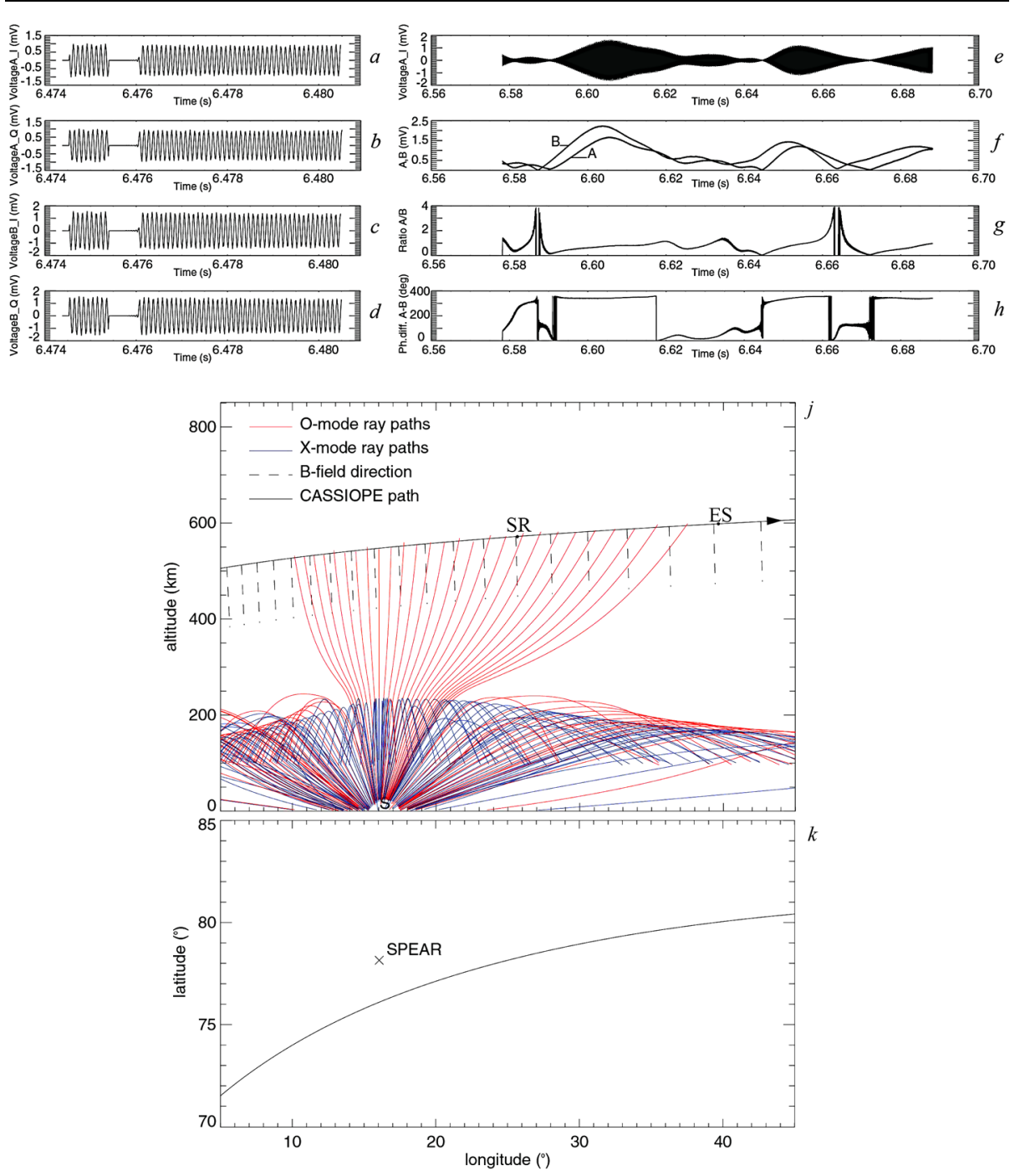

Fig. 17 Data from the RRI pass recorded starting at 15:34:06 UT on 17 Nov. 2013

\section{Early Mission Report on RRI}

Shortly after the insertion of CASSIOPE into orbit on 29 September 2013, the commissioning of the RRI began with the deployment of its four STEM jib monopoles. At about the same time, housekeeping data reports from the spacecraft confirmed that RRI was behaving as expected. Less than 2 days later, the first RRI scientific data were recorded: the RRI was commanded into a swept-frequency mode for the monopoles configured as dipoles, and the entire range of $30-\mathrm{kHz}$ band centers from $15 \mathrm{kHz}$ to about $18 \mathrm{MHz}$ swept. The examination of signal amplitudes showed noise bands associated with the characteristic electron frequencies up to about $5 \mathrm{MHz}$ and a few breakthrough signals from ground transmitters above $10 \mathrm{MHz}$ approximately. 
In addition to swept-frequency observations of spontaneous emissions, the RRI has detected ground transmitters at fixed frequency. One example of such fixed-frequency data is in Fig. 17. Parts $j$ and $k$ of this diagram show in continuous black line the CASSIOPE trajectory in altitude-longitude and latitude-longitude on 17 Nov. 2013 during the period 15:31:46 to 15:35:07 UT. Moving from left to right, the satellite transited the vicinity of the SPEAR ionospheric heater (Wright et al. 2000; UNIS 2014) at Longyearbyen, Svalbard, which was transmitting continuously at $4.45 \mathrm{MHz}$ with a left-hand polarization. The point "SR" on the path is the orbital position, at 15:34:06, when the RRI turned on. "ES" is the end-point of SPEAR signal detection by RRI, although RRI stayed on well past this point.

In panels $a$ through $d$ at the top left of Fig. 17, the I and Q components of the baseband voltage induced on the RRI dipoles A and B are plotted for the satellite location "SR". One short absence of signal aligned on tracks a-d indicates a data drop-out in the telemetry stream arising from its limited bandwidth. The similarity of signal amplitudes $a$ through $d$ confirms the correct deployment of the component monopoles. Panel $e$ shows the full amplitude wave form of dipole A on a slightly condensed time scale. In panel $f$ are plotted the amplitudes of the signal on both A and B dipoles. Dipole A is the dipole formed by monopoles 1 and 2, respectively at the top right and bottom left of Fig. 1.

Over an extended part of the pass of which this is a small section, but repeatedly over times less than $0.1 \mathrm{~s}$, the B dipole signal passed through zero. Apparently, B repeatedly passed through some fields where periodically B was perpendicular to the incoming wave electric field.

At Longyearbyen, an ionosonde measured a value of $f$ oF2 $=3.904 \mathrm{MHz}$ within $10 \mathrm{~min}$ utes of this pass. The corresponding $f \mathrm{xF} 2$ was therefore about 4.5 MHz. Making the large assumption that $f \mathrm{xF} 2$ was close to this value during the 1 minute of satellite pass and over the region of that pass depicted in Fig. 17, we infer that any X-mode rays, in blue, injected into the bottomside must have been reflected back earthward near the peak of the F2 layer. The radiation at the spacecraft then has the advantage, for propagation-direction determination, of being $\mathrm{O}$-mode only; there is no need to subtract $\mathrm{X}$-mode wave contributions. The ratio of the amplitudes of the A and B dipole signals, in panel $g$, and their phase difference, in panel $h$, can be used to deduce the O-mode wave vector direction at the spacecraft, with recourse to the cold-plasma polarization relations.

Unfortunately, the spacecraft was oriented for other purposes than for RRI dipole reception of these SPEAR transmissions. But other RRI-priority passes are being recorded in which the orientation of both dipoles point their bore sight roughly toward a source, and which therefore permit a measure of DOA.

\section{Concluding Remarks}

The design of RRI is predicated on the continuing need in e-POP to understand the microscale physics of processes occurring in localized regions of the ionosphere. Investigations on regional or even global scales of the ionosphere only succeed if there is adequate attention to the micro-scale descriptions that are the conceptual foundations of the larger-scale models. Also, technologies developed heretofore for measuring space plasmas do not have sufficient resolution to address small-scale structures being discovered in the ionosphere. The e-POP instrumentation answers this call for improved observations. Special applications of the RRI to in-situ active experiments show clearly the pertinence of this approach to space radio science. Electromagnetic wave observations and analysis remain an integral part of research 
on the physics of unbounded space plasmas, in particular the low density plasmas of the ionosphere-magnetosphere system.

It is hoped that, through the acquisition in Canada of a space-borne digital receiver, RRI will enhance other national opportunities to exercise and expand our expertise in highlatitude space plasmas. RRI exploits niche opportunities in Canada, and elsewhere, for multipoint investigations with ground and other space facilities. Indeed, international collaborations using the RRI and other e-POP instrumentation are being encouraged as a significant feature of the e-POP mission. RRI thereby helps to maintain Canadian expertise in spaceborne radio equipment.

Acknowledgements The financial support of the Canadian Space Agency, the valuable contribution of the COM DEV technical team responsible for the instrument's design and the technological expertise of the University of Calgary in the development of this instrument are all gratefully acknowledged.

Open Access This article is distributed under the terms of the Creative Commons Attribution License which permits any use, distribution, and reproduction in any medium, provided the original author(s) and the source are credited.

\section{References}

K.G. Balmain, Dipole admittance for magnetoplasma diagnostics. IEEE Trans. Antennas Propag. AP-17, 389-392 (1969)

K.G. Budden, The Propagation of Radio Waves-The Theory of Radio Waves of Low Power in the Ionosphere and Magnetosphere (Cambridge University Press, Cambridge, 1985)

H.G. James, High-frequency direction finding in the ionosphere. Rev. Sci. Instrum. 74(7), 3478-3486 (2003)

H.G. James, A Digital Radio Receiver for Ionospheric Research, in Characterising the Ionosphere. Meeting, Proceedings RTO-MP-IST-056, Paper 23. Neuilly-sur-Seine, France: RTO, (2006a), pp. 23-1-23-16. Available from: http://www.cso.nato.int/abstracts.aspx. ISBN 92-837-0078-3

H.G. James, Characteristics of field-aligned density depletion irregularities in the auroral ionosphere that duct Z- and X-mode waves. J. Geophys. Res. 111, A09315 (2006b). doi:10.1029/2006JA011652

H.G. James, C.L. Siefring, E.P. King, The Radio Receiver Instrument on the e-POP small satellite, in Conf. Proc., 12th Conf. on Astronautics Astro2002, CASI, Ottawa, Canada, 12-14 Nov. (2002)

UNIS, Space Plasma Exploration by Active Radar (SPEAR), The University Center of Svalbard, Longyearbyen, Norway, http://spear.unis.no (2014)

University of Calgary, e-POP Radio Receiver Instrument (RRI) User's Manual, Doc. no. ePOP-4370, Rev. D (2008a)

University of Calgary, e-POP Level 0 Data Description, Doc. no. ePOP-3523, Rev. A (2008b)

D.M. Wright et al., Space Plasma Exploration by Active Radar (SPEAR): An overview of a future facility. Ann. Geophys. 18, 1248-1255 (2000). doi:10.1007/s00585-000-1248-7

A.W. Yau, H.G. James, Scientific Objectives of the Canadian CASSIOPE Enhanced Polar Outflow Probe (e-POP) Small Satellite Mission, in The Sun, the Solar Wind, and the Heliosphere, ed. by M.P. Miralles, J.S. Almeida. IAGA Special Sopron Book Series, vol. 4 (2011), pp. 355-364. doi:10.1007/ 978-90-481-9787-3 